\title{
Comparación de cuatro técnicas quirúrgicas para el manejo de la espondilitis tuberculosa de la columna torácica en adultos
}

\author{
Comparison of four surgical techniques in the management of tuberculous spondylitis of \\ the thoracic spine in adults
}

\author{
Óscar A. Martínez-Gutiérrez, José E. Baena-Trejo, Víctor M. Peña-Martínez, Pedro M. Reyes-Fernández, \\ Elsa L. Zertuche-González, Francisco I. Villarreal-García y Rodolfo Morales-Avalos* \\ Servicio de Ortopedia y Traumatología, Hospital Universitario Dr. José Eleuterio González, Universidad Autónoma de Nuevo León, Monterrey, \\ Nuevo León, México
}

\begin{abstract}
Resumen
Objetivo: Comparar la eficiencia de las cuatro técnicas quirúrgicas más utilizadas para el manejo de la espondilitis tuberculosa. Método: Estudio retrospectivo en el que se incluyeron pacientes adultos con diagnóstico confirmado de espondilitis tuberculosa, afectación de dos niveles vertebrales o menos y sin deformidad vertebral grave. Se recopilaron y revisaron los expedientes médicos, los estudios de imagen y los datos demográficos de los pacientes intervenidos para analizar retrospectivamente los resultados clínicos y funcionales de cada grupo. Las variables primarias fueron la erradicación de la infección, la fusión vertebral y las complicaciones. Entre las variables secundarias se estudiaron el sangrado intraoperatorio, la estancia hospitalaria y el tiempo quirúrgico. Resultados: Entre los grupos analizados no hubo diferencias significativas $(p \geq 0.05)$ en la mayoría de las variables analizadas, pero sí $(p \leq 0.001)$ respecto al sangrado, el tiempo quirúrgico, la estancia intrahospitalaria y las complicaciones, a favor del abordaje posterior único. Conclusiones: El abordaje posterior único logró una eficacia clínica similar a la del resto de los abordajes en términos de erradicación de la infección y fusión vertebral; sin embargo, se asoció a menores tiempo quirúrgico, sangrado, estancia hospitalaria y complicaciones.
\end{abstract}

Palabras clave: Abordaje anterior. Abordaje combinado. Abordaje posterior. Columna torácica. Espondilitis tuberculosa. Tuberculosis espinal.

\begin{abstract}
Objective: To compare the efficiency of the 4 most used surgical techniques for the management of tuberculous spondylitis. Method: Retrospective study in which adult patients with a confirmed diagnosis of tuberculous spondylitis, involvement of two vertebral levels or less, and without severe vertebral deformity were included. The medical records, imaging studies, and demographic data of the operated patients were collected and reviewed to retrospectively analyze the clinical results of each group. The primary variables were cure of infection, spinal fusion, and complications. The secondary variables included intraoperative bleeding, hospital stay, and surgical time. Results: There were no significant differences $(p \geq 0.05)$ in most of the variables analyzed, however, there were $(p \leq 0.001)$ regarding bleeding, surgical time, hospital stay and complications between the
\end{abstract}

\section{Correspondencia:}

*Rodolfo Morales-Avalos

Avda. Francisco I. Madero s/n

Col. Mitras Centro

Fecha de recepción: 15-08-2020

Cir Cir. 2021;89(3):295-302

C.P. 64460, Monterrey, N.L., México

Fecha de aceptación: 07-10-2020

Contents available at PubMed

E-mail: rodolfot59@ hotmail.com

DOI: $10.24875 / \mathrm{CIRU} .20000907$

www.cirugiaycirujanos.com

0009-7411/@ 2020 Academia Mexicana de Cirugía. Publicado por Permanyer. Este es un artículo open access bajo la licencia CC BY-NC-ND (http://creativecommons.org/licenses/by-nc-nd/4.0/). 
groups analyzed, with a lower result in all cases for the single posterior approach. Conclusions: The single posterior approach obtained a clinical efficacy similar to the rest of the approaches in terms of eradication of the infection and vertebral fusion, however, it was associated with less surgical invasion (surgical time and bleeding), a shorter hospital stay and complications.

Key words: Anterior approach. Combined approach. Posterior approach. Thoracic spine. Tuberculous spondylitis. Spinal tuberculosis.

\section{Introducción}

La tuberculosis (TB) ha aumentado su prevalencia rápidamente en todo el mundo en los últimos años, especialmente en los países no desarrollados y en vías de desarrollo', como México, en particular en el noreste del país, donde su prevalencia es muy alta². La TB espinal es una de las presentaciones más comunes de TB extrapulmonar, y la forma más común y grave de presentación musculoesquelética, que comprende hasta el $50 \%$ de todos los pacientes afectados ${ }^{3}$. Esta causa destrucción de las columnas anterior y media, llevando a deformidad cifótica y déficit neurológico, entre otras complicaciones clínicas graves ${ }^{4}$.

La mayoría de los pacientes con TB espinal pueden ser tratados exitosamente con métodos conservadores, como un régimen de medicamentos orales ${ }^{5}$; sin embargo, algunos casos requieren intervenciones quirúrgicas. Las indicaciones para el tratamiento quirúrgico de la espondilitis tuberculosa son: 1) pacientes con déficit neurológico como consecuencia de la compresión de la médula espinal; 2) secuestro óseo y del disco intervertebral, así como presencia de cambios destructivos graves; 3) terapia convencional con medicación oral y ortesis externas no exitosa, con múltiples recurrencias; 4) inestabilidad significativa con deformidad espinal; 5) formación de abscesos epidurales $^{6} ;$ y 6) dolor intratable ${ }^{7}$. Los objetivos de la cirugía son erradicar la infección y prevenir o tratar el déficit neurológico mediante la eliminación de elementos compresivos (tejido necrótico y de granulación, hueso desvitalizado y material de disco), así como corregir la deformidad espinal. La cirugía también aumenta la vascularización, mejorando con ello la efectividad de los fármacos al aumentar su concentración en el sitio de la enfermedad, y por lo tanto ayuda en la curación y la fusión ósea ${ }^{8}$.

Las técnicas descritas para el tratamiento quirúrgico incluyen el desbridamiento anterior y la fusión intersomática con o sin instrumentación suplementada ${ }^{9}$, el desbridamiento posterior con instrumentación y el desbridamiento anterior con fusión intersomática combinada con instrumentación posterior en uno o dos tiem$\operatorname{pos}^{10}$. Se han reportado resultados favorables con el uso de todas estas técnicas; sin embargo, en algunos reportes se han apreciado ciertas diferencias con respecto a las variables intraoperatorias, los resultados clínicos posoperatorios y las complicaciones ${ }^{11}$. En la literatura consultada no hemos hallado ningún estudio que compare todas estas técnicas en un mismo reporte, pero sí se han reportado en otros estudios de manera independiente. La hipótesis de este estudio es que el desbridamiento y la instrumentación mediante un abordaje posterior es tan eficaz con respecto al desbridamiento y la instrumentación por un abordaje anterior y el abordaje combinado en uno o dos tiempos para el manejo de la TB de la columna torácica. El objetivo de este estudio fue comparar retrospectivamente la eficiencia de las cuatro técnicas mencionadas para el manejo de la espondilitis tuberculosa en la columna torácica en adultos.

\section{Método}

\section{Diseño del estudio y consideraciones éticas}

Este fue un estudio retrospectivo, transversal y descriptivo. Se siguieron las guías STROBE (Strengthening the Reporting of Observational studies in Epidemiology) para el reporte de estudios descriptivos retrospectivos. El estudio fue aprobado por el Comité de Ética en Investigación de nuestro hospital antes de ser realizado y está de acuerdo con la Declaración de Helsinki de la Asociación Médica Mundial de 1964 y su revisión de 2013 ${ }^{12}$. Los autores declaran que no existen ganancias financieras ni comerciales por la realización de este estudio, no tienen ningún conflicto de intereses y no recibieron financiamiento externo de ningún tipo.

\section{Criterios de elegibilidad}

Se incluyeron en el estudio pacientes mayores de 18 años con diagnóstico de espondilodiscitis tuberculosa activa de la columna torácica que fueron intervenidos quirúrgicamente en nuestra institución entre enero de 2002 y diciembre de 2019. El diagnóstico fue confirmado por cultivo, biopsia o reacción en 
cadena de la polimerasa cuantitativa (últimos 5 años), siguiendo en todos los casos el protocolo interno de nuestro hospital, el cual consiste en realizar una biopsia transpedicular bajo guía fluoroscópica y toma de muestras intraoperatorias. Otros criterios de inclusión fueron el compromiso de dos o menos vértebras adyacentes y la presencia de una deformidad espinal de tipo A según la clasificación de Rajasekaran ${ }^{13}$. Los criterios de exclusión fueron pacientes con cualquiera de las siguientes condiciones: afectación multinivel (más de dos vértebras adyacentes), TB de la columna cervical o lumbar, TB con afectación de los elementos posteriores, TB en múltiples niveles vertebrales no contiguos y aquellos pacientes que previamente se habían sometido a cirugía para el manejo de TB de la columna torácica o lumbar. Todos los casos fueron operados por el autor principal.

El abordaje inicial del paciente con sospecha de TB espinal fue clínico-radiológico. Se realizaron estudios de laboratorio (biometría hemática completa, velocidad de sedimentación globular [VSG], proteína C reactiva [PCR], pruebas de función hepática y renal, y prueba cutánea de la tuberculina [Mantoux]), radiografía de tórax, radiografías en vistas anteroposteriores y laterales de la columna dorsal y lumbar, tomografía computarizada y resonancia magnética de la columna toracolumbar. En todos los casos, los estudios se realizaron antes de iniciar un régimen cuádruple de quimioterapia antituberculosa con isoniazida (300 mg/día), rifampicina (450 mg/día), etambutol (750 $\mathrm{mg} / \mathrm{día}$ ) y pirazinamida (750 mg/día) por vía oral durante 2-4 semanas antes de la cirugía.

Se recopilaron y revisaron los expedientes médicos, los estudios de imagen y los datos demográficos de los pacientes intervenidos para analizar retrospectivamente los resultados clínicos de cada grupo. Las variables analizadas se clasificaron en seis tipos: 1) características clínicas, demográficas y de presentación de la enfermedad; 2) variables intraoperatorias y posoperatorias (pérdida de sangre, transfusión sanguínea, tiempo de cirugía, duración de la estancia hospitalaria, etc.); 3) corrección de la deformidad espinal, fusión (ángulo de Cobb, fusión radiológica según los criterios de Lee, et al..$^{14}$ ) y supresión de la infección (PCR $<0.6 \mathrm{mg} / \mathrm{dl}$ y VSG $<20 \mathrm{~mm} / \mathrm{h}$ en ausencia de patología inflamatoria sistémica o tumores malignos (según los criterios de Masuda, et al. ${ }^{15}$ ); 4) valores de laboratorio; 5) estado neurológico (utilizando la escala ASIA [American Spinal Injury Association]), preoperatorio y al final del seguimiento; y 6) complicaciones posoperatorias y mortalidad. Las variables primarias fueron la curación de la infección, la fusión vertebral y las complicaciones. Las variables secundarias fueron el sangrado intraoperatorio, la estancia hospitalaria, el tiempo quirúrgico, la corrección de la deformidad, el dolor y los valores de laboratorio.

\section{Indicaciones quirúrgicas}

Se indicó la cirugía si los pacientes tenían una o más de las siguientes condiciones: 1) presencia progresiva de déficit neurológico (paraplejía de grado Frankel A/B o paraplejía de grado Frankel C/D que no responde al manejo médico en $3-4$ semanas ${ }^{16} ; 2$ ) absceso epidural o formación de abscesos fríos; 3) más de un $50 \%$ de destrucción de los cuerpos vertebrales; 4) deformidad cifótica grave; 5) dolor intratable; y 6) infección persistente que no respondió al tratamiento conservador. La decisión del momento de llevar a cabo el procedimiento quirúrgico fue cuando la VSG y la temperatura corporal regresaron a los parámetros normales, o cuando habían disminuido significativamente, así como la corrección completa de la anemia y la hipoproteinemia o un aumento del déficit neurológico.

\section{Técnicas quirúrgicas}

Las técnicas quirúrgicas que se han empleado en nuestro centro y que se comparan en este estudio son: 1) desbridamiento radical anterior, fusión intersomática con injerto óseo autólogo e instrumentación anterior (grupo A); 2) desbridamiento posterior, fusión transforaminal e instrumentación posterior (grupo B); 3) abordaje combinado en un solo tiempo (instrumentación posterior más desbridamiento anterior y colocación de injerto óseo autólogo) (grupo C); y 4) abordaje combinado en dos tiempos (grupo D). Todos los procedimientos se realizaron bajo anestesia general e intubación endotraqueal.

\section{Grupo $A$ (abordaje anterior únICO)}

Se realizó desbridamiento radical anterior en un solo tiempo, fusión intersomática con injerto óseo autógeno/autólogo e instrumentación con placa bloqueada lateral. Los pacientes fueron colocados en decúbito lateral derecho y el área lesionada fue expuesta a través de un abordaje intrapleural realizado por un cirujano de tórax. La descompresión se realizó mediante corpectomía sola o corpectomía/disectomía. 
Posteriormente se realizó un desbridamiento radical del tejido afectado y se extirparon el tejido necrótico y el tejido de granulación restante hasta visualizar hueso sangrante sano. La instrumentación se llevó a cabo mediante una placa bloqueada lateral de titanio en $Z$ para corregir la deformidad cifótica, la cual se extendió un nivel por encima y un nivel por debajo de la zona afectada. El hueso obtenido de la resección de las costillas superiores en combinación con aloinjertos estructurales (diáfisis de tibia o peroné) se utilizó para el defecto resultante del desbridamiento radical.

\section{Grupo B (abordaje POSTERIOR ÚNICO)}

Se realizó una técnica de desbridamiento posterior en un solo tiempo, fusión transforaminal e instrumentación con tornillos transpediculares. Inicialmente, el paciente se colocó en decúbito prono con dos soportes acolchonados para el pecho y la pelvis. Se realizó una incisión en la línea media para exponer los elementos vertebrales y paravertebrales posteriores. Se realizó una disección para aislar las vértebras afectadas y dos niveles vertebrales por encima y por debajo. Los tornillos transpediculares se insertaron en las vértebras expuestas previamente bajo guía fluoroscópica. Se realizaron laminectomías en el nivel o niveles afectados con el fin de descomprimir y visualizar el cordón medular. Las apófisis espinosas, las láminas, las facetas articulares y las apófisis transversas en el área afectada fueron resecadas. Las costillas en el nivel superior se disecaron a $4 \mathrm{~cm}$ de la articulación costotransversa. Se realizó una ligadura cuidadosa de los vasos sanguíneos segmentarios y se desplazaron meticulosamente las raíces nerviosas de la zona expuesta. Después de que la zona lesionada fue completamente expuesta, el desbridamiento radical se llevó a cabo cuidadosamente con el fin de eliminar el tejido necrótico y de granulación hasta visualizar hueso sangrante sano. Además, cualquier absceso existente fue drenado. Después de realizado el desbridamiento radical, la cifosis resultante se corrigió mediante la instrumentación realizada. La reconstrucción de la columna vertebral se hizo colocando jaulas de malla de titanio rellenas de hueso esponjoso autólogo obtenido de la resección previa.

\section{Grupo C (abordaje combinado en Un tiempo)}

Se realizó un abordaje combinado en un solo tiempo con instrumentación posterior transpedicular seguido de un desbridamiento radical anterior. Inicialmente, la instrumentación posterior se aplicó para obtener una fijación suficiente; se colocaron tornillos pediculares en las dos vértebras superiores y las dos inferiores en relación con la afectada. Los niveles a fusionar fueron injertados por vía anterior utilizando una combinación de hueso autólogo y alogénico después de la decorticación de las láminas y las apófisis espinosas y transversas. Después de la instrumentación posterior, los pacientes fueron recolocados en decúbito lateral con el fin de realizar un abordaje anterior y eliminar el tejido infectado. La zona afectada fue aislada (esto incluía abscesos locales, vértebras colapsadas o discos intervertebrales) y se realizó el desbridamiento de tejido necrótico y de granulación, así como la extracción de pus hasta llegar a hueso sangrante sano. Después de lograr una descompresión suficiente, se utilizaron injertos estructurales autólogos para llenar el defecto creado en el proceso de desbridamiento y estabilizar la columna anterior.

\section{Grupo D (abordaje Posterior en dos tiempos)}

Se realizó un abordaje combinado en dos tiempos con instrumentación posterior seguido de un desbridamiento anterior. Se emplearon las mismas técnicas quirúrgicas que en el grupo $\mathrm{C}$, pero cada intervención se llevó a cabo en ocasiones separadas. En la primera ocasión (primera etapa) se realizó instrumentación posterior y se finalizó la cirugía. Dos a seis semanas después del procedimiento, según el estado de recuperación del paciente, se realizó una nueva intervención para realizar un desbridamiento radical anterior, así como la colocación de injertos óseos autólogos para rellenar el defecto.

\section{Cuidados posoperatorios y seguimiento}

En todos los casos se colocó un drenaje a succión que se retiró cuando el volumen fue menor de $50 \mathrm{ml}$ en 24 horas. Se permitió la ambulación asistida de 2 a 4 semanas después de la cirugía con la utilización de una faja dorso-lumbar. El seguimiento se realizó durante el primer año a las 6 semanas, los 3, 6 y 9 meses, y 1 año. Posteriormente se hicieron seguimientos de control con intervalos anuales. En cada consulta de seguimiento, los pacientes fueron evaluados clínicamente para valorar la función neurológica y el dolor, así como radiológicamente para la alineación de la 
columna vertebral y el grado de la fusión. En cada visita se obtuvieron radiografías anteroposteriores y laterales de la columna torácica, que se compararon con las obtenidas antes e inmediatamente después de la cirugía. La alineación sagital se evaluó con el método de Cobb. Se realizó una tomografía computarizada de la columna torácica para determinar el estado de fusión a los 6 meses y 1 año después de la cirugía. La monitorización de los parámetros de laboratorio (recuento de glóbulos blancos, VSG y PCR) se hizo regularmente hasta que los parámetros volvieron a los valores normales. Después de la cirugía, el régimen oral de quimioterapia antituberculosa se continuó durante 3 meses y luego se continuó con isoniazida y rifampicina hasta 12 meses.

\section{Análisis estadístico}

Se utilizó el programa estadístico SPSS versión 20.0 (IBM, Armonk, NY, USA). Para los datos cuantitativos se calcularon la media y la desviación estándar, y para las variables categóricas se expresaron frecuencias y porcentajes. Para el análisis inferencial se realizaron las pruebas $t$ de Student y ANOVA para las comparaciones de los grupos con variables numéricas paramétricas. La prueba de ji al cuadrado se empleó para analizar variables cualitativas entre grupos. Un valor de $p<0.05$ se consideró una diferencia estadísticamente significativa.

\section{Resultados}

Se incluyeron 48 pacientes, de los cuales 31 eran de sexo masculino $(64.58 \%)$ y 17 femenino $(35.42 \%)$. La edad media de los pacientes fue de $45 \pm 7.12$ años. La duración media de la enfermedad fue de $8 \pm 2.3$ meses.

Todos los pacientes sufrieron diversos grados de dolor, rigidez y limitación en la columna vertebral, y experimentaron un alivio inmediato de su dolor de espalda en el primer día de posoperatorio, con descenso de la fiebre después de varios días. Los síntomas experimentados por los pacientes preoperatoriamente incluían pérdida de peso, fiebre, sudoración nocturna y fatiga; los datos de laboratorio eran inespecíficos, como anemia, hipoproteinemia y elevación de la VSG y de la PCR. En los estudios de imagen, en todos los casos se encontraron datos consistentes con secuestros óseos, diseminación subligamentosa de la infección, destrucción y colapso del cuerpo vertebral, y formación de abscesos paraespinales.
No se observó deterioro neurológico tras la cirugía en ninguno de los casos. Todos los casos de TB espinal incluidos en el estudio se consideraron curados según los valores de laboratorio (PCR y VSG) y la evidencia radiológica de fusión y formación de puentes óseos en la tomografía computarizada y las radiografías simples.

En la tabla 1 se muestran los resultados de cada uno de los grupos incluidos en el presente estudio y la comparación estadística entre ellos. Puede verse que no existieron diferencias significativas $(p \geq 0.05)$ en la mayoría de las variables analizadas, pero sí las hubo ( $p \leq 0.001$ ) en cuanto al sangrado, el tiempo quirúrgico, la estancia hospitalaria y las complicaciones, con un resultado inferior en todos los casos para el grupo B (abordaje posterior único). La evolución posoperatoria fue favorable en todos los pacientes, pero con una tasa significativamente menor de complicaciones en el grupo con abordaje posterior único.

\section{Discusión}

En este análisis retrospectivo comparamos los resultados clínicos de cuatro técnicas quirúrgicas diferentes en el manejo de la espondilitis tuberculosa de la columna torácica en adultos. El principal hallazgo fue que el abordaje posterior único resulta ser tan eficaz como el resto de los abordajes, con una tasa significativamente menor de complicaciones y una disminución significativa del sangrado y de la estancia hospitalaria.

Los reportes en la literatura son muy escasos con respecto a este tema y se han publicado algunos estudios retrospectivos que analizan la experiencia de centros con cualquiera de las técnicas mencionadas; sin embargo, a nuestro conocimiento, no hay ningún estudio hasta la fecha que tenga como objetivo comparar la eficacia de todos estos abordajes entre sí, y consideramos que esta comparación es crucial para determinar el mejor procedimiento, teniendo en cuenta la eficacia y las complicaciones de cada uno.

Históricamente, los pacientes habían sido intervenidos mediante desbridamiento anterior con la colocación de injerto óseo, sobre todo debido a que el foco infeccioso se localiza en la parte anterior de la columna vertebral; por lo tanto, un abordaje anterior proporciona acceso directo a la zona infectada. Sin embargo, este abordaje tiene limitaciones importantes, de las cuales la más notable es la inestabilidad de la columna resultante de esta intervención, por lo que los pacientes permanecían postrados durante 
Tabla 1. Comparacion de los datos demográficos, clínicos y posoperatorios de los pacientes con los cuatro abordajes estudiados en el presente estudio

\begin{tabular}{|c|c|c|c|c|c|}
\hline Variable & Grupo A $(n=12)$ & Grupo $B(n=12)$ & Grupo $C(n=14)$ & Grupo $D(n=10)$ & p \\
\hline Sexo (H: M) & $8: 4$ & $8: 4$ & $9: 5$ & $6: 4$ & - \\
\hline Edad (años) & $47.92 \pm 8.16$ & $42.54 \pm 7.16$ & $50.15 \pm 5.78$ & $48.56 \pm 4.79$ & 0.067 \\
\hline Pérdida del cuerpo vertebral (\%) & $0.44 \pm 0.04$ & $0.41 \pm 0.06$ & $0.48 \pm 0.05$ & $0.40 \pm 0.06$ & 0.129 \\
\hline Seguimiento (meses) & $32.33 \pm 16.24$ & $28.62 \pm 11.13$ & $24.46 \pm 6.79$ & $32.39 \pm 4.45$ & 0.212 \\
\hline Nivel vertebral más afectado (moda) & T10-T11 (9/12) & T9-T10 (9/12) & T9-T10 (11/14) & T10-T11 (8/10) & - \\
\hline $\begin{array}{l}\text { Escala ASIA (deterioro neurológico) } \\
\text { preoperatoria }\end{array}$ & $4 \mathrm{E}, 5 \mathrm{D}, 2 \mathrm{C}, 1 \mathrm{D}$ & $5 E, 4 D, 3 C$ & $10 \mathrm{E}, 3 \mathrm{D}, 1 \mathrm{C}$ & $7 \mathrm{E}, 3 \mathrm{D}$ & - \\
\hline Escala ASIA (deterioro neurológico) al año & $9 \mathrm{E}, 2 \mathrm{D}, 1 \mathrm{C}$ & $9 \mathrm{E}, 3 \mathrm{D}$ & $12 \mathrm{E}, 2 \mathrm{D}$ & $9 \mathrm{E}, 1 \mathrm{D}$ & - \\
\hline Sangrado quirúrgico $(\mathrm{ml})$ & $680 \pm 314$ & $512 \pm 245$ & $985 \pm 477$ & $900 \pm 412$ & $<0.001$ \\
\hline Tiempo de la cirugía (min) & $176 \pm 37.44$ & $160 \pm 23.12$ & $410 \pm 60.45$ & $386 \pm 78.45$ & $<0.001$ \\
\hline Estancia hospitalaria (días) & $14.9 \pm 4.23$ & $10.5 \pm 2.25$ & $22.3 \pm 5.8$ & $18.9 \pm 4.77$ & $<0.001$ \\
\hline Mortalidad (n, \%) & $0 \%$ & $0 \%$ & $0 \%$ & $1(10 \%)$ & - \\
\hline Tiempo para la fusión (meses) & $6.2 \pm 1.22$ & $6.5 \pm 1.41$ & $6.7 \pm 1.02$ & $6.0 \pm 1.51$ & 0.657 \\
\hline Fusión alcanzada (\%) & $91.67 \%(11 / 12)$ & $100 \%$ & $100 \%$ & $100 \%$ & - \\
\hline PCR preoperatoria $(\mathrm{mg} / \mathrm{l})$ & $14.69 \pm 7.35$ & $15.86 \pm 6.45$ & $19.45 \pm 8.45$ & $18.89 \pm 3.11$ & 0.257 \\
\hline PCR posoperatoria, 3 meses (mg/l) & $5.77 \pm 2.89$ & $6.45 \pm 3.12$ & $5.54 \pm 2.22$ & $5.51 \pm 2.37$ & 0.811 \\
\hline PCR posoperatoria, 1 año (mg/l) & $0.45 \pm 0.19$ & $0.58 \pm 0.29$ & $0.63 \pm 0.14$ & $0.65 \pm 0.25$ & 0.102 \\
\hline VSG preoperatoria $(\mathrm{mm} / \mathrm{h})$ & $78.54 \pm 15.89$ & $50.23 \pm 12.7$ & $55.15 \pm 18.45$ & $61.3 \pm 11.44$ & 0.124 \\
\hline VSG posoperatoria, 3 meses (mm/h) & $18.5 \pm 5.89$ & $22.78 \pm 6.78$ & $15.0 \pm 5.7$ & $17.8 \pm 5.89$ & 0.061 \\
\hline VSG posoperatoria, 1 año (mm/h) & $13.44 \pm 3.89$ & $15.98 \pm 3.57$ & $8.44 \pm 2.34$ & $11.67 \pm 3.99$ & 0.875 \\
\hline Curación de la infección (\%) & $100 \%$ & $100 \%$ & $100 \%$ & $100 \%$ & - \\
\hline Cobb preoperatorio (grados) & $19.56 \pm 4.56$ & $23.29 \pm 4.19$ & $30.45 \pm 8.47$ & $27.1 \pm 5.78$ & 0.067 \\
\hline Cobb posoperatorio, 3 meses (grados) & $4.6 \pm 0.87$ & $5.17 \pm 0.68$ & $8.79 \pm 1.43$ & $6.24 \pm 1.17$ & 0.061 \\
\hline Cobb posoperatorio, 1 año (grados) & $6.98 \pm 0.89$ & $6.14 \pm 0.75$ & $6.14 \pm 1.98$ & $6.99 \pm 2.02$ & 0.315 \\
\hline EVA preoperatoria & $8.5 \pm 2.15$ & $7.12 \pm 1.11$ & $8.33 \pm 1.55$ & $7.88 \pm 1.10$ & 0.140 \\
\hline EVA posoperatoria, 3 meses & $2.78 \pm 1.54$ & $2.23 \pm 1.31$ & $3.01 \pm 1.14$ & $2.69 \pm 1.00$ & 0.478 \\
\hline EVA posoperatoria, 1 año & $1.5 \pm 0.86$ & $1.02 \pm 0.52$ & $1.55 \pm 0.69$ & $1.33 \pm 0.78$ & 0.263 \\
\hline Absceso epidural (n, \%) & $2(16.67 \%)$ & $3(25 \%)$ & $1(7.14 \%)$ & $1(10 \%)$ & 0.598 \\
\hline Absceso paravertebral (n, \%) & $8(66.67 \%)$ & $9(75 \%)$ & $10(71.42 \%)$ & $6(60 \%)$ & 0.885 \\
\hline Pérdida de la corrección (grados) & $1.89 \pm 1.06$ & $1.12 \pm 0.89$ & $1.10 \pm 0.22$ & $1.00 \pm 0.56$ & 0.121 \\
\hline Corrección del ángulo (grados) & $14.2 \pm 3.86$ & $16.99 \pm 4.56$ & $17.19 \pm 5.85$ & $18.12 \pm 5.33$ & 0.278 \\
\hline Complicaciones ( $\mathrm{n}, \%)$ & $\begin{array}{c}2(16.67 \%)(1 \\
\text { hemotórax y } 1 \\
\text { derrame pleural) }\end{array}$ & $0(0 \%)$ & $\begin{array}{c}2(14.28 \%)(1 \\
\text { neuralgia intercostal y } \\
1 \text { neumonía) }\end{array}$ & $\begin{array}{c}3 \text { (30\%) (1 fístula LCR, } \\
1 \text { neumotórax y } 1 \\
\text { infección profunda) }\end{array}$ & $<0.001$ \\
\hline
\end{tabular}

ASIA: American Spinal Injury Association; EVA: escala visual análoga; H: hombre; LCR: líquido cefalorraquídeo; M: mujer; PCR: proteína C reactiva; VSG: velocidad de sedimentación globular.

largos periodos de tiempo y corrían el riesgo de no unión, cifosis residual o aparición de úlceras por decúbito. Además, este procedimiento solo es adecuado para un cierto número de niveles vertebrales, por lo que sus indicaciones son notablemente limitadas. Aunque el uso combinado de una instrumentación por 
vía anterior ha aumentado la eficacia de esta vía y ha disminuido sus complicaciones, es bien conocido que realizar una instrumentación por vía anterior en la columna torácica es complicado por la cercanía topográfica de estructuras anatómicas vitales y el área limitada de trabajo $0^{17,18}$.

Un abordaje anterior y posterior combinado es muy útil para disminuir la pérdida de estabilidad relacionada con el abordaje anterior único, por lo que se ha convertido en una opción quirúrgica popular para el manejo de la espondilitis tuberculosa. Algunos autores mencionan que este tipo de abordaje se prefiere en los casos en que hay una rigidez grave en la columna torácica y el abordaje por una sola vía sería insuficiente $^{19}$. El abordaje posterior único ha sido criticado porque se argumenta que por una vía posterior resultaría difícil lograr un desbridamiento adecuado de la porción anterior del cuerpo vertebral; sin embargo, los estudios han determinado que mediante un abordaje extendido con costotransversectomía añadida puede realizarse de manera adecuada y colocar el injerto correctamente para lograr una fusión a largo plazo 20,21 .

Zeng, et al. ${ }^{22}$ realizaron un estudio con 39 pacientes con diagnóstico de TB espinal toracolumbar, de los cuales un grupo fue operado por abordaje posterior único y otro por abordaje combinado. Encontraron que el abordaje posterior único disminuía significativamente el tiempo quirúrgico, el sangrado y las complicaciones, sin incrementar los índices de fusión y erradicación de la infección. Zhang, et al.. ${ }^{23}$, en un estudio retrospectivo en el que compararon el abordaje posterior único frente al abordaje combinado en el manejo de pacientes ancianos con espondilitis tuberculosa de la columna torácica, encontraron que el abordaje posterior obtuvo mejores resultados clínicos que el abordaje combinado.

\section{Limitaciones}

Este estudio tiene algunas limitaciones, pues aunque se trate de una muestra amplia para una patología rara, el número de pacientes sigue siendo bajo y está distribuido en cuatro grupos. Del mismo modo, la naturaleza retrospectiva del estudio representa una limitación por sí misma, y un seguimiento a más largo plazo sería ideal para establecer conclusiones definitivas. Reconocemos que, al tratarse de un estudio retrospectivo, la elección del tratamiento, el tipo de implantes y la técnica de fijación/estabilización no son homogéneos y no se siguió un proceso de aleatorización

\section{Conclusiones}

El abordaje posterior único obtuvo una eficacia clínica similar a la del resto de los abordajes en términos de erradicación de la infección y fusión vertebral, pero se asoció a una menor invasión quirúrgica (tiempo quirúrgico y sangrado), una menor estancia hospitalaria y una menor tasa de complicaciones, por lo que consideramos que es un abordaje seguro y eficaz para el manejo de la espondilitis tuberculosa en adultos. Aun así, son necesarios más estudios con mayor número de pacientes y un seguimiento más largo para establecer conclusiones definitivas.

\section{Responsabilidades éticas}

Protección de personas y animales. Los autores declaran que para esta investigación no se han realizado experimentos en seres humanos ni en animales.

Confidencialidad de los datos. Los autores declaran que han seguido los protocolos de su centro de trabajo sobre la publicación de datos de pacientes.

Derecho a la privacidad y consentimiento informado. Los autores han obtenido el consentimiento informado de los pacientes y/o sujetos referidos en el artículo. Este documento obra en poder del autor de correspondencia.

\section{Financiamiento}

Recursos propios del departamento de los autores.

\section{Conflicto de intereses}

Los autores declaran no tener conflictos de intereses.

\section{Bibliografía}

1. World Health Organization. Global tuberculosis report 2019. Geneva: World Health Organization; 2019. Licence: CC BY-NC-SA 3.0 IGO.

2. Zazueta-Beltran J, León-Sicairos $C$, Canizalez-Román A. Drug resistant Mycobacterium tuberculosis in Mexico. J Infect Dev Ctries. 2009;3:162-8.

3. Kaloostian PE, Gokaslan ZL. Current management of spinal tuberculosis: a multimodal approach. World Neurosurg. 2013;80:64-5.

4. Al-Sebai MW, Al-Khawashki H, Al-Arabi K, Khan F. Operative treatment of progressive deformity in spinal tuberculosis. Int Orthop. 2001;25:322-5.

5. Dimar JR, Carreon LY, Glassman SD, Campbell MJ, Hartman MJ, Johnson JR. Treatment of pyogenic vertebral osteomyelitis with anterior debridement and fusion followed by delayed posterior spinal fusion. Spine (Phila Pa 1976). 2004;29:326-32. 
6. Buyukbebeci O, Karakurum G, Guleç A, Erbagci A. Tuberculous osteomyelitis of the lumbosacral region: a spinal epidural abscess with presacral extension. Arch Orthop Trauma Surg. 2004;124:346-8.

7. Sahoo MM, Mahapatra SK, Sethi GC, Dash SK. Posterior-only approach surgery for fixation and decompression of thoracolumbar spinal tuberculosis: a retrospective study. J Spinal Disord Tech. 2012;25: E217-23

8. Ge Z, Wang Z, Wei M. Measurement of the concentration of three antituberculosis drugs in the focus of spinal tuberculosis. Eur Spine $\mathrm{J}$. 2008;17:1482-7.

9. Yilmaz C, Selek HY, Gürkan I, Erdemli B, Korkusuz Z. Anterior instrumentation for the treatment of spinal tuberculosis. J Bone Joint Surg Am. 1999;81:1261-7.

10. Dai LY, Jiang LS, Wang W, Cui YM. Single-stage anterior autogenous bone grafting and instrumentation in the surgical management of spinal tuberculosis. Spine (Phila Pa 1976). 2005;30:2342-9.

11. Klöckner C, Valencia R. Sagittal alignment after anterior debridement and fusion with or without additional posterior instrumentation in the treatment of pyogenic and tuberculous spondylodiscitis. Spine (Phila $\mathrm{Pa}$ 1976). 2003;28:1036-42.

12. World Medical Association. World Medical Association Declaration of Helsinki: ethical principles for medical research involving human subjects. JAMA. 2013;310:2191-4.

13. Rajasekaran S. The natural history of post-tubercular kyphosis in children. Radiological signs which predict late increase in deformity. J Bone Joint Surg Br. 2001;83:954-62.

14. Lee CK, Vessa P, Lee JK. Chronic disabling low back pain syndrome caused by internal disc derangements. The results of disc excision and posterior lumbar interbody fusion. Spine (Phila Pa 1976). 1995;20:356-61.
15. Masuda T, Miyamoto K, Hosoe H, Shimizu K. Comparative study on the efficacy of two-staged (posterior followed by anterior) surgical treatment using spinal instrumentation on pyogenic and tuberculotic spondylitis. Arch Orthop Trauma Surg. 2011;131:765-72

16. Frankel HL, Hancock DO, Hyslop G, Melzak J, Michaelis LS, Ungar GH, et al. The value of postural reduction in the initial management of closed injuries of the spine with paraplegia and tetraplegia. I. Paraplegia. 1969;7:179-92.

17. Li M, Du J, Meng $\mathrm{H}$, Wang Z, Luo Z One-stage surgical management for thoracic tuberculosis by anterior debridement, decompression and autogenous rib grafts, and instrumentation. Spine J. 2011;11:726-33.

18. Benli IT, Kaya A, Acaroğlu E. Anterior instrumentation in tuberculous spondylitis: is it effective and safe? Clin Orthop Relat Res. 2007:460:108-16.

19. Wang Z, Yuan H, Geng G, Shi J, Jin W. Posterior mono-segmental fixation, combined with anterior debridement and strut graft, for treatment of the mono-segmental lumbar spine tuberculosis. Int Orthop. 2012;36:325-9.

20. Kumar MN, Joseph B, Manur R. Isolated posterior instrumentation for selected cases of thoraco-lumbar spinal tuberculosis without anterior instrumentation and without anterior or posterior bone grafting. Eur Spine J. 2013;22:624-32

21. Yang $P, \mathrm{He} X, \mathrm{Li} \mathrm{H}$, Zang $Q$, Yang B. Clinical efficacy of posterior versus anterior instrumentation for the treatment of spinal tuberculosis in adults: a meta-analysis. J Orthop Surg Res. 2014:9:10.

22. Zeng $\mathrm{H}$, Wang $\mathrm{X}$, Pang $\mathrm{X}$, Luo $\mathrm{C}$, Zhang $\mathrm{P}$, Peng $\mathrm{W}$, et al. Posterior only versus combined posterior and anterior approaches in surgical management of lumbosacral tuberculosis with paraspinal abscess in adults. Eur J Trauma Emerg Surg. 2014:40:607-16.

23. Zhang HQ, Li JS, Zhao SS, Shao YX, Liu SH, Gao Q, et al. Surgical management for thoracic spinal tuberculosis in the elderly: posterior only versus combined posterior and anterior approaches. Arch Orthop Trauma Surg. 2012;132:1717-23. 